\title{
High Mean Corpuscular Volume as a Predictor of Poor Overall Survival in Patients with Esophageal Cancer Receiving Concurrent Chemoradiotherapy
}

This article was published in the following Dove Press journal: Cancer Management and Research

\author{
Ke-jie $\mathrm{Li}^{1, *}$ \\ Wen-yue $\mathrm{Gu}^{2, *}$ \\ Xiao-fang Xia' \\ Ping Zhang ${ }^{3}$ \\ Chang-lin Zou (iD ${ }^{3}$ \\ Zheng-hua $\mathrm{Fei}^{3}$ \\ 'Department of Radiation Oncology, The \\ First Affiliated Hospital of Wenzhou \\ Medical University, Wenzhou, 325000 , \\ People's Republic of China; ${ }^{2}$ Department \\ of Pathology, Yancheng Hospital Affiliated \\ of Southeast University, Yancheng, Jiangsu \\ 224000, People's Republic of China; \\ ${ }^{3}$ Radiation and Medical Oncology \\ Department, The First Affiliated Hospital \\ of Wenzhou Medical University, \\ Wenzhou 325000, People's Republic of \\ China
}

*These authors contributed equally to this work
Correspondence: Chang-lin Zou; Zhenghua Fei

Department of Radiation Oncology, The First Affiliated Hospital of Wenzhou Medical University, No. 2 Fuxue Lane, Wenzhou 325000, People's Republic of China

Tel +86-I37587366II; +86-I5868728796

Fax +86-577-88069292

Email zcl13705775570@।63.com;

feizhenghua@wzhospital.cn
Background: Increasing numbers of recent studies have demonstrated that high mean corpuscular volume (MCV) is a predictor of poor overall survival (OS) and therapeutic response in patients with solid tumors. The aim of the present study was to explore the association between high MCV and OS in patients with advanced esophageal cancer (EC) undergoing concurrent chemoradiotherapy.

Patients and Methods: Enrolled in this study were 249 patients with advanced EC who underwent concurrent chemoradiotherapy. Pre-treatment MCV values were collected in all patients and their correlations with OS and pathophysiological characteristics were analyzed. The chi-square test was used to explore the correlation between MCV and various clinical pathophysiological characteristics, and the prognostic significance of high MCV using Kaplan-Meier curves and the Cox proportional hazards model. All $P$-values were twotailed and a $P$-value $<0.05$ was considered statistically significant.

Results: According to ROC curve analysis, the optimal cut-off value of MCV was $93.6 \mathrm{fL}$. The mean OS was 14.7 months in all 249 EC patients, 10.9 months in patients with MCV $>93.6 \mathrm{fL}$, and 18.8 months in patients with $\mathrm{MCV}<93.6 \mathrm{fL}$; the difference is statistically significant $(\mathrm{P}<0.05)$. Chi-square test showed that the MCV value was correlated with the $\mathrm{N}$ stage of the tumor and the therapeutic effect, indicating that the higher the MCV was, the higher the $\mathrm{T}$ stage of the tumor and the worse the therapeutic effect would be $(\mathrm{p}=0.012$ and $\mathrm{p}<0.01)$. Multivariate analysis showed that MCV (OR $=1.864,95 \%$ CI: $1.439-2.415)$ was an independent prognostic factor for OS in EC patients.

Conclusion: High MCV is a poor predictor of OS in patients with advanced EC receiving concurrent chemoradiotherapy.

Keywords: esophageal cancer, mean corpuscular volume, concurrent chemoradiotherapy, predictors, overall survival

\section{Introduction}

Mean corpuscular volume (MCV) is one of the most common clinical hematological parameters. It refers to the mean volume of a single red blood cell in the human body. MCV is commonly used to assist in the diagnosis of hematological diseases; for instance, an increase in the mean red blood cell (RBC) volume is more common in large cell anemia, while a decrease in the mean RBC volume is more common in small cell anemia such as iron deficiency anemia and thalassemia. The predictive role of $\mathrm{MCV}$ in various solid tumors has received increasing attention in recent years. Motohiko Kato and his team reported that MCV was negatively correlated with the risk of advanced colorectal cancer (CRC). ${ }^{1}$ Nagai et al considered 
preoperative $\mathrm{MCV}$ as a prognostic factor for CRC and reported that patients with lower MCV had better diseasefree survival (DFS). ${ }^{2}$ Dellapasqua et al showed that MCV was a predictor of advanced metastatic breast cancer. ${ }^{3}$ Wang and her team reported that preoperative MCV was a predictive factor for patients with esophageal cancer (EC). ${ }^{4}$ And in a previously published retrospective study, postoperative MCV was a predictor of esophageal cancer survival. ${ }^{5}$ However, the correlation between $\mathrm{MCV}$ and overall survival (OS) after concurrent chemoradiotherapy in patients with advanced EC has not been reported.

Esophageal cancer is one of the most common global malignant tumors. According to the latest statistics, the incidence and mortality rates of EC rank the 7th and 6th in all malignant tumors, respectively. There will be about 580,000 new cases and 500,000 deaths around the world in 2018. ${ }^{6}$ Esophageal squamous cell carcinoma (ESCC) accounts for about $90 \% \mathrm{EC}$ and is the main pathological type. ${ }^{7}$ The development of EC is very secretive and rapid. Most patients are already in the advanced stage and lose the opportunity of surgery at the time of diagnosis. At present, the standard treatment for inoperable EC patients is local radiotherapy combined with systemic chemotherapy. ${ }^{8}$ According to statistics, about $80 \%$ patients with advanced EC received concurrent chemoradiotherapy. The Radiation Therapy Oncology Group (RTOG) 85-01 study reported that concurrent chemoradiotherapy could improve OS of patients with advanced unresectable EC, though the 5-year survival rate was still less than $30 \%{ }^{9}$ However, OS varies significantly with individual patients who receive the same concurrent chemoradiotherapy regimen. It is therefore particularly important to predict the survival possibility of each patient after treatment so as to help clinicians plan individualized treatment options for the sake of achieving the optimal outcome for ESCC patients. Wen and his team showed that miRNA could predict the response to concurrent chemoradiotherapy in EC patients. ${ }^{10}$ Butof et al reported that the pre-therapeutic tumor-to-blood Standardized Uptake Ratio (SUR) could predict the survival of EC patients. ${ }^{11}$ Chi et al showed that TRIM24 was a potential predictor of survival in EC patients. $^{12}$ The research by Wen $\mathrm{J}$ et al suggested that pretreatment gene expression analysis could help predict the response to concurrent chemoradiotherapy in ESCC patients, thus facilitating individualized treatment of tumor patients. ${ }^{13}$ However, the acquisition of these genes and factors requires accurate detection instruments and complex detection procedures, which are difficult to obtain in general hospitals. Given the previous studies on the predictive power of $\mathrm{MCV}$ in multiple solid tumors and the simplicity of $\mathrm{MCV}$ acquisition, our work aimed to investigate whether high MCV was a predictor of poor prognosis for patients with advanced EC receiving concurrent chemoradiotherapy.

\section{Patients and Methods}

\section{Patient Selection}

A total of 249 patients with advanced EC who received concurrent chemoradiotherapy in the first affiliated hospital of Wenzhou Medical University (Wenzhou, China) from 2013 to 2017 were enrolled in our study. Inclusion criteria are as follows: (1) patients aged 18-80 years; (2) patients with a histological diagnosis of ESCC; (3) ESCC patients who only received concurrent chemoradiotherapy after the diagnosis; (4) patients with no major blood diseases such as severe anemia, hypoproteinemia, malignant lymphoma and polycythemia eukaryotica at the time of diagnosis; (5) patients with complete follow-up data.

\section{Data Acquisition}

Hematological and routine biochemical indexes of all 249 patients were collected, including red RBC, white blood cell (WBC), platelet count, albumin (ALB), hemoglobin $(\mathrm{Hb}), \mathrm{RBC}$ distribution width (RDW), MCV, hematocrit (HCT), carcinoembryonic antigen (CEA), squamous cell carcinoma antigen (SCC-Ag), and carbohydrate antigen (CA) 19-9. In addition, basic tumor characteristics including location, size, degree of tumor differentiation and lymph node (LN) metastasis, and the baseline information of the patients including age, gender, smoking index, drinking history and ECOG score were also collected.

\section{Treatment Methods}

All patients received three-dimensional (3D) conformal therapy combined with systemic chemotherapy. 95\% PTVp and 95\% PTVnd received 60Gy in 30 fractions (on work days; 2 Gy per fraction; over a 6-week cycle). After all patients received CT localization to fully understand the size and range of the tumor, the target area was delineated by the senior physicians from the department of radiotherapy and chemotherapy. The criteria for delineating the target area are as follows: gross tumor volume 
(GTV), which refers to the primary esophageal lesion determined by the esophagogram display and/or the tumor size shown by esophagoscopy and/or intraventricular ultrasound combined with various imaging data; GTVnd, which refers to metastatic supraclavicular and paratracheal LN detected either by CT scan or palpation; and CTV, which refers to GTV, GTVnd and their LN drainage area and was placed around GTV and GTVnd for $0.8 \mathrm{~cm}$, put $3-5 \mathrm{~cm}$ above and below GTV and GTVnd. PTV is the external radiation of $0.5-1 \mathrm{~cm}$ on the basis of CTV, and the scope of PTV is appropriately released according to the specific situation of patients, so as to ensure that CTV can get the required prescription dose. The chemotherapy regimen for systemic chemotherapy was $5-\mathrm{Fu} 750 \mathrm{mg} / \mathrm{m}^{2}$ combined with cisplatin $75 \mathrm{mg} / \mathrm{m}^{2}$ once every four weeks. The study was approved by the ethics committee of the first affiliated hospital of Wenzhou Medical University. As all the patients included in this retrospective study have died, we sought the consent of the patients' immediate family members or the authorized persons who signed the informed consent before treatment and signed the written informed consent before the study.

\section{Assessment Strategies}

The primary endpoint of the study was OS of the EC patients, which refers to the time from randomization to (any cause) death. The secondary endpoints were progression-free survival (PFS) and the objective response rate (ORR). PFS was defined as the time between the initiation of randomization and tumor progression (in any respect) or death (for any reason). ORR refers to the proportion of tumor shrinkage reaching a certain volume and remaining for a certain period of time, including the total number of patients with complete response (CR) and partial response (PR). According to Response Evaluation Criteria In Solid Tumor (RECIST), CR was defined as the disappearance of all target lesions, the occurrence of no new lesions, and the short diameter of all pathological lymph nodes (including target and non-target nodules) reduced to $<10 \mathrm{~mm}$. PR was defined as reducing the sum of the diameters of target disease areas by at least 30\% from baseline. OS was obtained by telephone interviews by the clinicians. PFS and ORR were obtained through outpatient and inpatient cases and imaging data at review to guarantee the objective authenticity of the evaluation endpoints.

\section{Data Analysis}

All statistical analyses were performed using the statistical package for social science software program version 22.0 (SPSS Inc., Chicago, IL, USA). The receiver operating characteristic (ROC) curve was used to determine the optimal diagnostic value of each indicator. Univariate COX regression was performed to identify factors associated with OS and PFS. Multivariate COX regression analysis was performed to determine adverse factors associated with OS and PFS. Chi-square test was used to analyze the differences in clinicopathological characteristics. Kaplanmeier method was used to draw the survival curve, and $\mathrm{P}$ value $<0.05$ was considered statistically significant.

\section{Results}

The follow-up period ranged from 2 to 77 months with a median of 11 months. Of the 249 EC patients, 46 were women and 203 were men, with a mean age of 65.8 years. Patients with cervical and upper thoracic EC were assigned to group Upper esophageal cancer $(n=124)$, and those with middle and lower thoracic EC $(n=125)$ to group Lower esophageal cancer. Similarly, patients with moderately and highly differentiated EC were assigned to well-differentiated group ( $\mathrm{n}=180)$, and those with poorly differentiated EC to poor differentiated group $(n=69)$. The specific physiopathological characteristics of the patients are shown in Table 1.

The mean MCV value of the 249 patients was 92.7 fL. According to the analysis of ROC curve, the area under the curve (AUC) was 0.733, and the optimal diagnostic value of MCV was 93.6 fL (Figure 1). Chisquare test was used to evaluate differences in the pathophysiological characteristics between the two groups of MCV patients. The results showed that the MCV value was correlated with the $\mathrm{N}$ stage of the tumor and the therapeutic effect, indicating that the higher the MCV was, the higher the $\mathrm{T}$ stage of the tumor and the worse the therapeutic effect would be $(\mathrm{p}=0.012$ and $\mathrm{p}<0.01)$. In the group with $\mathrm{MCV}<93.6 \mathrm{fL}, 67$ patients achieved effective remission, accounting for $26.9 \%$ of all participants. In the group with MCV $>93.6 \mathrm{fL}$, only 51 patients achieved effective remission, accounting for $20.5 \%$ of the total subjects. However, no correlation was observed with other indicators, which may be related to the characteristics of the tumor (Table 2).

Univariate and multivariate cox analyses were used to explore whether MCV was an independent predictor of OS in 
Table I Basic Physiological and Physiological Characteristics of 249 Patients

\begin{tabular}{|c|c|}
\hline Characteristics & No. of People (\%) \\
\hline No of people & 249 \\
\hline \multicolumn{2}{|l|}{ Sex } \\
\hline Female & 203 \\
\hline Male & 46 \\
\hline \multicolumn{2}{|l|}{ Age } \\
\hline Median & 65.8 years old \\
\hline Range & $38-85$ years old \\
\hline 65 years old or older & 127 \\
\hline Under 65 years old & 122 \\
\hline \multicolumn{2}{|l|}{ History of smoking } \\
\hline Smoking & 126 \\
\hline No smoking & 123 \\
\hline \multicolumn{2}{|l|}{ Drinking history } \\
\hline Drinking & 124 \\
\hline No drinking & 125 \\
\hline \multicolumn{2}{|l|}{ Differentiation } \\
\hline Well differentiated & 180 \\
\hline Poor differentiated & 69 \\
\hline \multicolumn{2}{|l|}{ Tumor site } \\
\hline Upper esophageal cancer & 125 \\
\hline Lower esophageal cancer & 124 \\
\hline \multicolumn{2}{|l|}{ Tumor length (cm) } \\
\hline Median & $4.8 \mathrm{~cm}$ \\
\hline Range & $0.9-11.3 \mathrm{~cm}$ \\
\hline More than $5 \mathrm{~cm}$ & 123 \\
\hline Less than $5 \mathrm{~cm}$ & 126 \\
\hline \multicolumn{2}{|l|}{ Tumor width $(\mathrm{cm})$} \\
\hline Median & $3.6 \mathrm{~cm}$ \\
\hline Range & $0.7-8.9 \mathrm{~cm}$ \\
\hline More than $4 \mathrm{~cm}$ & 116 \\
\hline Less than $4 \mathrm{~cm}$ & 133 \\
\hline \multicolumn{2}{|l|}{ T-staging } \\
\hline $\mathrm{TI}+\mathrm{T} 2$ & 86 \\
\hline $\mathrm{T} 3+\mathrm{T} 4$ & 163 \\
\hline \multicolumn{2}{|l|}{$N$-staging } \\
\hline No & 159 \\
\hline $\mathrm{N} 1+\mathrm{N} 2$ & 90 \\
\hline \multicolumn{2}{|l|}{ ECOG score } \\
\hline 0 Point & 126 \\
\hline I Point & 82 \\
\hline 2 Point & 41 \\
\hline
\end{tabular}

patients with advanced EC. Median OS for all ESCC patients was 14.7 months (95\% CI 13.1-16.2 months). Univariate analysis showed that MCV, platelet count, the history of

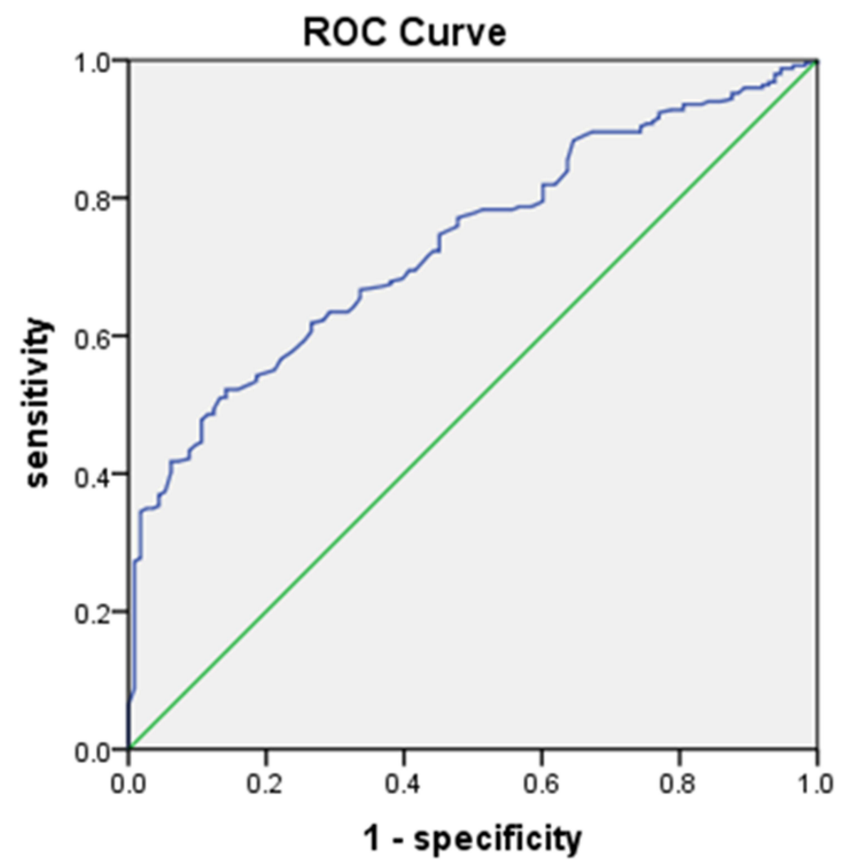

Figure I Receiver operating characteristic (ROC) curve plotted to check the value of a statistically significant variable in the COX regression model for MCV. According to ROC analysis, the areas under the curve of MCV was 0.733 and the optimal cutoff points was 93.6.

alcohol consumption, $\mathrm{N}$ stage, tumor width, and SCC-Ag were associated with OS of EC patients (Table 3). These factors were also observed to be associated with PFS. According to the multivariate survival analysis, MCV was an independent predictor of OS (Table 4), demonstrating that patients with a pretreatment MCV level $>93.6 \mathrm{fL}$ (the optimal diagnostic value for patients) had significantly shorter OS than those with a MCV level lower than $93.6 \mathrm{fL}$ (median OS 10.9 months vs. 18.8 months; $\mathrm{P}<0.05$ ) (Figure 2). These factors were also observed to be associated with the PFS. Patients who had a pretreatment MCV level $>93.6 \mathrm{fL}$ (the optimal diagnostic value for patients) had significantly shorter PFS than patients who had MCV level $<93.6 \mathrm{fL}$ (median Progress-Free Survival, 6.2 months vs. 13.5 months; $\mathrm{P}<0.05$ )(Figure 3).

\section{Discussion}

Esophageal cancer is one of the most common global malignant tumors. Currently available treatments for EC mainly include surgery, chemotherapy, radiotherapy, targeted therapy and immunotherapy. ${ }^{14}$ Concurrent chemoradiotherapy is the main method for the treatment of advanced EC, and survival of such patients after concurrent chemoradiotherapy has always been a focus of concern and attention. At present, TNM staging is the most 
Table 2 Univariate COX Regression Analysis of the Relationship Between Pathophysiological Parameters and Survival Time of Patients

\begin{tabular}{|c|c|c|c|}
\hline Parameter & OR & $95 \% \mathrm{Cl}$ & $\mathbf{P}$ \\
\hline Sex & 1.053 & $0.763-1.453$ & 0.752 \\
\hline Age & 1.082 & $0.843-1.390$ & 0.536 \\
\hline Smoking history & 1.227 & $0.953-1.580$ & 0.112 \\
\hline Drinking history & 1.308 & $1.015-1.688$ & 0.038 \\
\hline Differentiation & $0.88 I$ & $0.667-1.165$ & 0.374 \\
\hline Tumor site & 0.933 & $0.724-1.202$ & 0.592 \\
\hline Tumor length & 1.182 & $0.920-1.517$ & 0.191 \\
\hline Tumor width & 1.396 & $1.082-1.80 \mid$ & 0.010 \\
\hline T-staging & 1.153 & $0.886-1.500$ & 0.289 \\
\hline $\mathrm{N}$-staging & 1.531 & $1.174-1.997$ & 0.002 \\
\hline ECOG score & 1.166 & $0.833-1.633$ & 0.371 \\
\hline Leukocyte & 1.021 & $0.792-1.317$ & 0.870 \\
\hline Erythrocyte & 0.828 & $0.576-1.191$ & 0.309 \\
\hline Platelet & 1.297 & $1.003-1.678$ & 0.047 \\
\hline Hemoglobin & 0.967 & $0.693-1.349$ & 0.842 \\
\hline Albumin & 0.951 & $0.708-1.277$ & 0.737 \\
\hline Hematocrit & 0.996 & $0.77 \mid-1.286$ & 0.974 \\
\hline $\mathrm{MCV}$ & 1.966 & $1.525-2.536$ & $<0.01$ \\
\hline RDW & 0.908 & $0.700-1.176$ & 0.464 \\
\hline PCT & 0.820 & $0.634-1.061$ & 0.132 \\
\hline SCC & 1.431 & $1.041-1.967$ & 0.027 \\
\hline CA199 & 1.133 & $0.875-1.467$ & 0.343 \\
\hline CEA & 0.917 & $0.68 I-1.235$ & 0.570 \\
\hline
\end{tabular}

Table 3 Multivariate COX Regression Analysis of the Relationship Between Clinical Variables and Patient Survival

\begin{tabular}{|l|l|l|l|}
\hline Parameter & OR & $95 \% \mathbf{C l}$ & $\mathbf{P}$ \\
\hline Drinking history & 1.290 & $0.997-1.669$ & 0.052 \\
Tumor width & 1.249 & $0.964-1.620$ & 0.093 \\
N-staging & 1.433 & $1.092-1.880$ & 0.009 \\
MCV & 1.864 & $1.439-2.415$ & $<0.01$ \\
Platelet & 1.130 & $0.870-1.469$ & 0.359 \\
SCC & 1.190 & $0.858-1.650$ & 0.297 \\
\hline
\end{tabular}

effective predictor for survival of EC patients. ${ }^{15,16}$ However, there are significant differences in OS between patients with the same TNM staging receiving the same treatment. In addition, accurate TNM staging often requires postoperative pathological evaluation. It is therefore difficult to use TNM staging to predict survival and determine further treatment strategies for inoperable patients with advanced EC. Other effective predictors are required urgently for such patients.

MCV is a relatively stable blood indicator in healthy people. With the advent of automatic blood counting, the
Table 4 Relationship Between Pretreatment MCV and Clinicopathological Parameters in Patients with ESCC Who Received Concurrent Radiochemotherapy

\begin{tabular}{|c|c|c|c|}
\hline Characteristic, n=204 & $M C V<93.6$ & $M C V>93.6$ & $\mathbf{P}$ \\
\hline \multicolumn{4}{|l|}{ Sex } \\
\hline Female & 23 & 23 & \\
\hline Male & 95 & 108 & 0.745 \\
\hline \multicolumn{4}{|l|}{ Age } \\
\hline$<65$ years old & 59 & 63 & \\
\hline$>65$ years old & 59 & 68 & 0.800 \\
\hline \multicolumn{4}{|l|}{ Smoking history } \\
\hline Smoking & 54 & 72 & \\
\hline No smoking & 64 & 59 & 0.164 \\
\hline \multicolumn{4}{|l|}{ Drinking history } \\
\hline Drinking & 56 & 68 & \\
\hline No drinking & 62 & 63 & 0.527 \\
\hline \multicolumn{4}{|l|}{ Tumor site } \\
\hline Upper & 62 & 63 & \\
\hline Lower & 56 & 68 & 0.527 \\
\hline \multicolumn{4}{|l|}{ Differentiation } \\
\hline Well & 85 & 95 & \\
\hline Poor & 33 & 36 & 0.522 \\
\hline \multicolumn{4}{|l|}{ Tumor length } \\
\hline$>5 \mathrm{~cm}$ & 55 & 68 & \\
\hline$<5 \mathrm{~cm}$ & 63 & 63 & 0.447 \\
\hline \multicolumn{4}{|l|}{ Tumor width } \\
\hline$>4 \mathrm{~cm}$ & 51 & 65 & \\
\hline$<4 \mathrm{~cm}$ & 67 & 66 & 0.373 \\
\hline \multicolumn{4}{|l|}{ T-staging } \\
\hline $\mathrm{TI}+\mathrm{T} 2$ & 45 & 41 & \\
\hline $\mathrm{T} 3+\mathrm{T} 4$ & 73 & 90 & 0.287 \\
\hline \multicolumn{4}{|l|}{$\mathrm{N}$-staging } \\
\hline No & 85 & 74 & \\
\hline $\mathrm{N} 1+\mathrm{N} 2$ & 33 & 57 & 0.012 \\
\hline \multicolumn{4}{|l|}{ ECOG score } \\
\hline 0 Point & 98 & 110 & \\
\hline IPoint+2Point & 20 & 21 & 0.866 \\
\hline \multicolumn{4}{|l|}{ Curative effect } \\
\hline$C R+P R$ & 67 & 35 & \\
\hline SD+PD & 51 & 96 & $<0.01$ \\
\hline
\end{tabular}

clinical significance of its increase and decrease in predicting a diseased condition has attracted increasing attention. In addition to being used to assist in the diagnosis of hematological diseases, the clinical significance of MCV has also been reported in other diseases. Solak et al reported a possible relationship between MCV and cardiovascular 


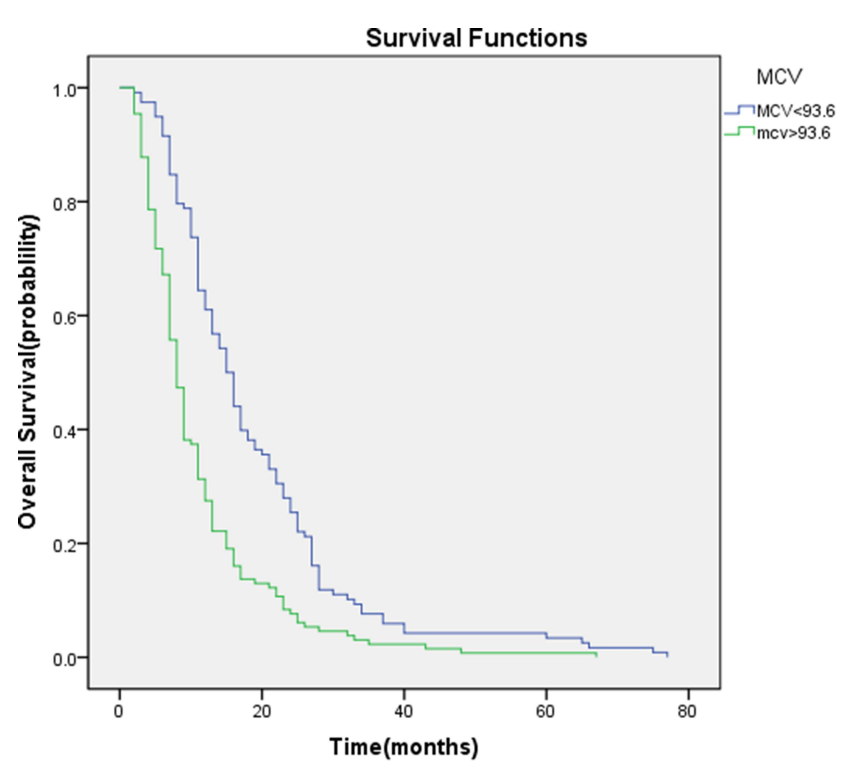

Figure 2 Kaplan-Meier survival curves for advanced esophageal cancer in different MCV groups. The blue curve represents the overall survival of the group with MCV $<93.6 \mathrm{fL}$, while the green curve represents the overall survival of the group with $\mathrm{MCV}>93.6 \mathrm{fL}$. The mean survival time of patients in the group with $\mathrm{MCV}<93.6 \mathrm{fL}$ was 10.9 months, while that of patients in the group with $\mathrm{MCV}>93.6 \mathrm{fL} 18.8$ months, $P<0.05$, indicating a significant difference between the two groups.

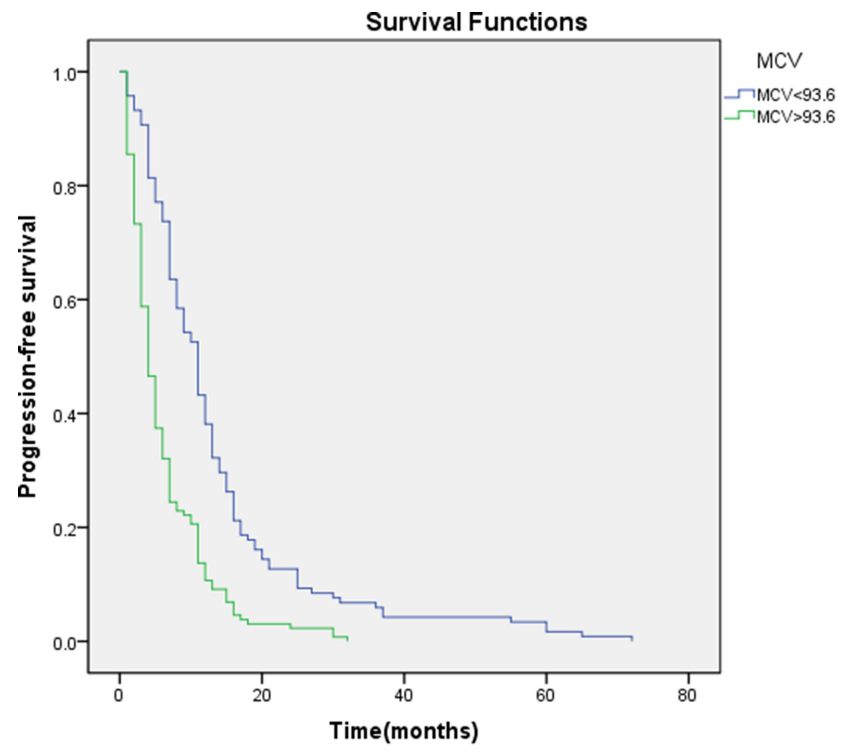

Figure 3 Kaplan-Meier survival curves describe the relationship between progression-free survival time and MCV in different groups. In the $\mathrm{MCV}<93.6 \mathrm{fL}$ group, the mean progression-free survival time was 13.5 months. For $M C V>93.6 \mathrm{fL}$, mean progression-free survival time was 6.2 months, $P<0.05$, indicating a significant difference between the two groups.

events in patients with chronic kidney disease. ${ }^{17}$ Yoon et al reported that MCV could be used as an independent survival predictor for healthy populations. ${ }^{18}$ Many other studies have also reported the relationship between MCV and survival of patients with solid tumors. Jomrich and his team found that high MCV was a poor prognostic factor for esophageal adenocarcinoma. ${ }^{19} \mathrm{Qu}$ and his team showed that MCV was associated with survival of patients with resectable non-small cell lung cancer (NSCLC). ${ }^{20}$ Our study has demonstrated that high MCV is a poor predictor of survival in patients with advanced EC after concurrent chemoradiotherapy.

In this study, we analyzed the relationship between MCV and survival in patients with advanced EC who received radiotherapy and chemotherapy. First, we used the ROC curve to analyze and determine the optimal cutoff value of $\mathrm{MCV}$, finding that the optimal cut-off value of MCV was 93.6 fL. Univariate and multivariate COX regression analyses showed that MCV was closely correlated with survival of the patients with advanced EC who received concurrent chemoradiotherapy. The analysis showed that OS of patients with a high level of MCV before treatment was significantly shorter than that of patients with a low-level MCV, showing a negative correlation between them. When we used PFS as a secondary endpoint, we obtained the same result. Meanwhile, the chi-square test analysis showed that MCV was related to the $\mathrm{N}$ stage of the patient and the therapeutic effect. This means that the size of MCV may be associated with lymph node metastasis.

Our study on the relationship between MCV and solid tumors have proved that high MCV is a poor prognostic factor but were unable to confirm whether MCV plays a direct role in local recurrence and metastatic invasion of tumors. We have several hypotheses as to why high $\mathrm{MCV}$ is a poor predictor of advanced EC. First, MCV is an important marker of the folate concentration in the body, and folate deficiency often leads to increased MCV. Folic acid, as an important carbon unit transfer carrier in the body, plays an indispensable role in DNA synthesis, replication, repair and methylation. Folate deficiency can lead to abnormal DNA methylation, which is a poor predictor of prognosis in EC patients. ${ }^{21,22}$ Second, previous studies have shown that survival of EC patients is related to the individual's basic nutritional status. ${ }^{23,24}$ Progressive dysphagia is the main clinical symptom of EC patients. ${ }^{25}$ In patients with advanced esophageal cancer, reduced food intake reduces the serum concentration of $\mathrm{Na}+, \mathrm{K}+$ and other electrolytes, leading to the reduction in plasma crystal osmotic pressure. The size of MCV in the internal environment of the human body is negatively correlated with the osmotic pressure of plasma crystals, and decreased osmotic pressure of plasma crystals increases $\mathrm{MCV}^{26}$ Moreover, according to the definition of MCV, we can know that the size of MCV value is related to the 
hematocrit per liter of blood and the number of RBCs per liter of blood. Increase in the MCV value may be due to the decrease of RBCs per unit volume of blood, and RBCs are involved in oxygen transport and metabolism of the body. The decrease in the number of RBCs will lead to a decrease in the amount of oxygen that the tumor tissue gets from the circulating blood, which will lead to a decrease in the total oxygen content in the tumor tissue and an increase in the proportion of hypoxic tumor cells. Hypoxia will lead to a decrease in the sensitivity of tumor cells to radiotherapy, which will greatly reduce the therapeutic effect and lead to poor prognosis. ${ }^{27-30}$ Finally, drug resistance is likely to occur after the use of chemotherapy drugs. However, previous studies demonstrated that $\mathrm{MCV}$ was increased after the administration of capecitabine and other drugs, so whether high MCV is an insensitivity factor of chemotherapy and thus affects the prognosis of patients is also something that we need to explore. $^{31}$

Although our data and results were confirmed and calculated repeatedly, this study has some limitations. Firstly, the number of patients in this study is only 249 , which may lead to unstable results due to the small sample size. Secondly, because the study was a retrospective study, we could not control all possible risk factors of cancer patients, and our data were all from medical documents, and registration of these medical documents could not be completely accurate due to the busy clinical work. Therefore, when collecting patient data, our team hopes to reduce the error to the minimum after repeated verification and statistics by many people. Furthermore, it was a single-center retrospective study that included all patients from the same hospital, and its conclusions were not verified by other centers. Therefore, further prospective trials in multiple centers are required to confirm the repeatability of these results in heterogeneous populations. Finally, this study only demonstrated that high MCV was an adverse predictor for EC patients, but how to use specific MCV to judge patient survival in clinical practice requires further research and the establishment of a more systematic prediction model.

\section{Conclusion}

High mean corpuscular volume as a predictor of poor overall survival in patients with esophageal cancer receiving concurrent chemoradiotherapy. In our clinical treatment of newly diagnosed advanced esophageal cancer patients, we can use MCV to roughly predict their survival. For those patients whose survival is predicted to be short, we can adopt a relatively conservative treatment method to reduce the cycle of chemoradiotherapy, thus reducing the toxic side effects of chemotherapy on patients. And for those patients who predict long survival, we can use more aggressive treatment to achieve the best treatment results.

\section{Abbreviations}

$\mathrm{MCV}$, mean corpuscular volume; OS, overall survival; EC, esophageal cancer; ROC curve, receiver operating characteristic curve; OR, odds ratio; $\mathrm{CI}$, confidence interval; $\mathrm{RBC}$, red blood cell; CRC, colorectal cancer; ESCC, esophageal squamous cell cancer; RTOG, Radiation Therapy Oncology Group; SUR, standardized Uptake Ratio; WBC, white blood cell; ALB, albumin; Hb, hemoglobin; RDW, Red Cell volume Distribution Width; HCT, hematocrit; CEA, carcinoembryonic antigen; SCC-Ag, squamous cell carcinoma antigen; CA19-9, carbohydrate antigen 19-9; LN, lymph node; CT, Computed Tomography; GTV, gross tumor volume; CTV, clinical target volume; PTV, Planning Target Volume; PFS, progression-free survival; ORR, objective response rate; $\mathrm{CR}$, complete response; $\mathrm{PR}$, partial response.

\section{Ethics Approval and Consent to Participate}

All the procedures followed were in accordance with the ethical guidelines of the Helsinki Declaration. The study protocol was approved by the Medical Ethical Committee of the First Affiliated Hospital of Wenzhou Medical University. All patients' family were given informed written consent.

\section{Acknowledgments}

This study was supported by grant from the Natural Science Foundation of Zhejiang Province, China (No. LY15H160063) and Wenzhou Science and Technology Bureau Foundation (grant no.2019Y0355). Ke-Jie Li and Wen-yue $\mathrm{Gu}$ are joint first authors for this study.

\section{Disclosure}

The authors declare that they have no competing interests.

\section{References}

1. Kato M, Kubosawa Y, Hiarai Y, et al. Usefulness of mean corpuscular volume for detection of advanced colorectal cancer in patients older than 85 years. Digestion. 2018;97(2):177-182. doi:10.1159/000485 378 
2. Nagai H, Yuasa N, Takeuchi E, et al. The mean corpuscular volume as a prognostic factor for colorectal cancer. Surg Today. 2018;48 (2):186-194. doi:10.1007/s00595-017-1575-x

3. Dellapasqua S, Bagnardi V, Bertolini F, et al. Increased mean corpuscular volume of red blood cells predicts response to metronomic capecitabine and cyclophosphamide in combination with bevacizumab. Breast. 2012;21(3):309-313. doi:10.1016/j.breast.2012.01.015

4. Zheng Y-Z, Dai SQ, Li W, et al. Prognostic value of preoperative mean corpuscular volume in esophageal squamous cell carcinoma. World J Gastroenterol. 2013;19(18):2811-2817. doi:10.3748/wjg.v19.i18.2811

5. Yoshida N, Kosumi K, Tokunaga R, et al. Clinical importance of mean corpuscular volume as a prognostic marker after esophagectomy for esophageal cancer: a retrospective study. Ann Surg. 2018.

6. Bray F, Ferlay J, Soerjomataram I, et al. Global cancer statistics 2018: GLOBOCAN estimates of incidence and mortality worldwide for 36 cancers in 185 countries. CA Cancer J Clin. 2018;68 (6):394-424. doi:10.3322/caac.21492

7. Arnold M, Soerjomataram I, Ferlay J, et al. Global incidence of oesophageal cancer by histological subtype in 2012. Gut. 2015;64 (3):381-387. doi:10.1136/gutjnl-2014-308124

8. van Rossum PSN, Mohammad NH, Vleggaar FP, et al. Treatment for unresectable or metastatic oesophageal cancer: current evidence and trends. Nat Rev Gastroenterol Hepatol. 2018;15(4):235-249. doi:10.1038/nrgastro.2017.162

9. Cooper JS, Guo MD, Herskovic A, et al. Chemoradiotherapy of locally advanced esophageal cancer: long-term follow-up of a prospective randomized trial (RTOG 85-01). Radiation Therapy Oncology Group. JAMA. 1999;281(17):1623-1627. doi:10.1001/ jama.281.17.1623

10. Wen J, Luo K, Liu H, et al. MiRNA expression analysis of pretreatment biopsies predicts the pathological response of esophageal squamous cell carcinomas to neoadjuvant chemoradiotherapy. Ann Surg. 2016;263(5):942-948. doi:10.1097/SLA.0000000000001489

11. Butof R, Hofheinz F, Zophel K, et al. Prognostic value of pretherapeutic tumor-to-blood standardized uptake ratio in patients with esophageal carcinoma. $J$ Nucl Med. 2015;56(8):1150-1156. doi:10.2967/jnumed.115.155309

12. Chi J, Yang Q, Xie X-F, et al. Clinical significance and prognostic value of TRIM24 expression in esophageal squamous cell carcinoma. Aging. 2016;8(9):2204-2221. doi:10.18632/aging.101037

13. Wen J, Yang H, Liu MZ, et al. Gene expression analysis of pretreatment biopsies predicts the pathological response of esophageal squamous cell carcinomas to neo-chemoradiotherapy. Ann Oncol. 2014;25 (9):1769-1774. doi:10.1093/annonc/mdu201

14. Klevebro F, Ekman S, Nilsson M. Current trends in multimodality treatment of esophageal and gastroesophageal junction cancer review article. Surg Oncol. 2017;26(3):290-295. doi:10.1016/j. suronc.2017.06.002

15. Brown CS, Gwilliam N, Kyrillos A, et al. Predictors of pathologic upstaging in early esophageal adenocarcinoma: results from the national cancer database. The American Journal of Surgery. 2018;216(1):124-130. doi:10.1016/j.amjsurg.2017.07.015

16. Jiao X, Krasna MJ, Sonett J, et al. Pretreatment surgical lymph node staging predicts results of trimodality therapy in esophageal cancer. Eur J Cardiothorac Surg. 2001;19(6):880-886. doi:10.1016/S10107940(01)00737-0

Cancer Management and Research

\section{Publish your work in this journal}

Cancer Management and Research is an international, peer-reviewed open access journal focusing on cancer research and the optimal use of preventative and integrated treatment interventions to achieve improved outcomes, enhanced survival and quality of life for the cancer patient.
17. Solak Y, Yilmaz MI, Saglam M, et al. Mean corpuscular volume is associated with endothelial dysfunction and predicts composite cardiovascular events in patients with chronic kidney disease. Nephrology. 2013;18(11):728-735. doi:10.1111/nep.12130

18. Yoon H-J, Kim K, Nam Y-S, et al. Mean corpuscular volume levels and all-cause and liver cancer mortality. Clin Chem Lab Med. 2016;54(7):1247-1257. doi:10.1515/cclm-2015-0786

19. Jomrich G, Hollenstein M, John M, et al. High mean corpuscular volume predicts poor outcome for patients with gastroesophageal adenocarcinoma. Ann Surg Oncol. 2019;26(4):976-985. doi:10. 1245/s10434-019-07186-1

20. Qu X, Zhang T, Ma H, et al. Lower mean corpuscular hemoglobin concentration is associated with unfavorable prognosis of resected lung cancer. Future Oncol. 2014;10(14):2149-2159. doi:10.2217/fon.14.121

21. Kim Y-I. Will mandatory folic acid fortification prevent or promote cancer? Am J Clin Nutr. 2004;80(5):1123-1128. doi:10.1093/ajcn/ 80.5.1123

22. Sato F, Meltzer SJ. CpG island hypermethylation in progression of esophageal and gastric cancer. Cancer. 2006;106(3):483-493. doi: $10.1002 /$ encr.21657

23. World MJ, Ryle PR, Jones D, et al. Differential effect of chronic alcohol intake and poor nutrition on body weight and fat stores. Alcohol Alcohol. 1984;19(4):281-290.

24. Ruka W, Rutkowski P, Kaminska J, et al. Alterations of routine blood tests in adult patients with soft tissue sarcomas: relationships to cytokine serum levels and prognostic significance. Ann Oncol. 2001;12(10):1423-1432. doi:10.1023/A:1012527006566

25. Sami S, Haboubi HN, Ang Y, et al. UK guidelines on oesophageal dilatation in clinical practice. Gut. 2018;67(6):1000-1023. doi:10.11 36/gutjnl-2017-315414

26. A A. The mutual effect of hydrogen ion concentration and osmotic pressure on the shape of the human erythrocyte as determined by light scattering and by electronic cell volume measurement. Cytometry. 1983;4(3):263-267. doi:10.1002/cyto.990040312

27. Achermann R, Ohlerth S, Fidel J, et al. Ultrasound guided, pre-radiation oxygen measurements using polarographic oxygen needle electrodes in spontaneous canine soft tissue sarcomas. In vivo (Athens, Greece). 2002;16(6):431-437.

28. Brunner-Agten S, von Känel T, Röthlisberger B, et al. Hb bakersfield (HBA1: c.151_152insGGAGCC): the insertion of arg-his between codons 49 and 50 of the $\alpha 1$-globin chain leads to increased oxygen affinity. Hemoglobin. 2017;41(1):1-5. doi:10.1080/03630269.2017. 1302467

29. Reiss UM, Bensimhon P, Zimmerman SA, et al. Hydroxyurea therapy for management of secondary erythrocytosis in cyanotic congenital heart disease. Am J Hematol. 2007;82(8):740-743. doi:10.1002/ ajh.20925

30. O'Donnell JL, Joyce MR, Shannon AM, et al. Oncological implications of hypoxia inducible factor- $1 \alpha$ (HIF-1 $\alpha$ ) expression. Cancer Treat Rev. 2006;32(6):407-416. doi:10.1016/j.ctrv.2006.05.003

31. Scarabelli L, Giovanardi F, Gervasi E, et al. Increased mean corpuscular volume of red blood cells in patients treated with capecitabine for advanced breast and colon cancer. Chemotherapy. 2013;59 (5):369-372. doi:10.1159/000357771
The manuscript management system is completely online and includes a very quick and fair peer-review system, which is all easy to use. Visit http://www.dovepress.com/testimonials.php to read real quotes from published authors.

\section{Dovepress}

